\title{
AN ALGEBRAIC, ENERGY CONSERVING FORMULATION OF CLASSICAL MOLECULAR AND NEWTONIAN $n$-BODY INTERACTION
}

\author{
BY DONALD GREENSPAN
}

Communicated by Fred Brauer, August 14, 1972

1. Introduction. In this note we will develop a unifying theory of collisionless $n$-body problems which includes both Newtonian and classical molecular forces. By using only differences to simulate physical concepts and difference equations to determine dynamical behavior, the resulting theory will be completely arithmetic in nature. Thus, we will have the advantages of mathematical simplicity and computer compatibility. The formulation will have special value for problems involving large amounts of energy, since it will be energy conserving.

2. Basic concepts. For positive time step $\Delta t$, let $t_{k}=k \Delta t, k=0,1,2, \ldots$. At time $t_{k}$, let particle $P_{i}$ of mass $m_{i}$ be located at $\boldsymbol{x}_{i, k}=\left(x_{i, k}, y_{i, k}, z_{i, k}\right)$, have velocity $\boldsymbol{v}_{i, k}=\left(v_{i, k, x}, v_{i, k, y}, v_{i, k, z}\right)$, and have acceleration $\boldsymbol{a}_{i, k}=$ $\left(a_{i, k, x}, a_{i, k, y}, a_{i, k, z}\right)$, for $i=1,2, \ldots, n$. Position, velocity, and acceleration are assumed to be related by the fundamental formulas [2]:

$$
\begin{aligned}
\left(\boldsymbol{v}_{i, k+1}+\boldsymbol{v}_{i, k}\right) / 2 & =\left(\boldsymbol{x}_{i, k+1}-\boldsymbol{x}_{i, k}\right) /(\Delta t), \\
\boldsymbol{a}_{i, k} & =\left(\boldsymbol{v}_{i, k+1}-\boldsymbol{v}_{i, k}\right) /(\Delta t) .
\end{aligned}
$$

If $\boldsymbol{F}_{i, k}=\left(F_{i, k, x}, F_{i, k, y}, F_{i, k, z}\right)$ is the force acting on $P_{i}$ at time $t_{k}$, then force and acceleration are assumed to be related by the discrete dynamical equation

$$
\boldsymbol{F}_{i, k}=m_{i} \boldsymbol{a}_{i, k} .
$$

The work $W_{i}$ done by $\boldsymbol{F}_{i, k}$ on $P_{i}$ from initial time $t_{0}$ to terminal time $t_{N}$ is defined by

$$
W_{i}=\sum_{k=0}^{N-1}\left[\left(\boldsymbol{x}_{i, k+1}-\boldsymbol{x}_{i, k}\right) \cdot \boldsymbol{F}_{i, k}\right],
$$

while the total work $W$ done on the system from time $t_{0}$ to time $t_{N}$ is defined by

$$
W=\sum_{i=1}^{n} W_{i}
$$

AMS(MOS) subject classifications (1970). Primary 70F10, 39A10. 
3. Conservation of kinetic energy. From (2.1)-(2.4), it follows that

$$
\begin{aligned}
W_{i} & =m_{i} \sum_{k=0}^{N-1}\left[\left(x_{i, k+1}-x_{i, k}\right) a_{i, k, x}+\left(y_{i, k+1}-y_{i, k}\right) a_{i, k, y}+\left(z_{i, k+1}-z_{i, k}\right) a_{i, k, z}\right] \\
& =\frac{1}{2} m_{i} \sum_{k=0}^{N-1}\left[\left(v_{i, k+1, x}^{2}-v_{i, k, x}^{2}\right)+\left(v_{i, k+1, y}^{2}-v_{i, k, y}^{2}\right)+\left(v_{i, k+1, z}^{2}-v_{i, k, z}^{2}\right)\right] \\
& =\frac{1}{2} m_{i}\left(v_{i, N, x}^{2}+v_{i, N, y}^{2}+v_{i, N, z}^{2}\right)-\frac{1}{2} m_{i}\left(v_{i, 0, x}^{2}+v_{i, 0, y}^{2}+v_{i, 0, z}^{2}\right) .
\end{aligned}
$$

Thus, if the kinetic energy $K_{i, k}$ of $P_{i}$ at $t_{k}$ is defined by

$$
K_{i, k}=\frac{1}{2} m_{i}\left(v_{i, k, x}^{2}+v_{i, k, y}^{2}+v_{i, k, z}^{2}\right),
$$

then

$$
W_{i}=K_{i, N}-K_{i, 0} .
$$

Finally, if the kinetic energy $K_{k}$ of the system at $t_{k}$ is defined by

$$
K_{k}=\sum_{i=0}^{n} K_{i, k}
$$

then (2.5), (3.2), and (3.3) imply

$$
W=K_{N}-K_{0},
$$

which is called the law of conservation of kinetic energy.

It is interesting to note that (3.4) is valid independently of the specific structure of the forces involved.

4. The $n$-body force law. In order to simulate Newtonian gravitation and various classical laws of molecular interaction $([\mathbf{1}],[\mathbf{5}])$, we will structure the force between each pair of particles to consist of a component of attraction which behaves like $p /\left(r^{\alpha}\right)$ and a component of repulsion which behaves like $q /\left(r^{\beta}\right)$, where $p, q, \alpha$, and $\beta$ are nonnegative parameters and $r$ is the distance between the particles, as follows. Let $r_{i j, k}$ be the distance between $P_{i}$ and $P_{j}$ at time $t_{k}$. Then $\boldsymbol{F}_{i, k}$, the force exerted on $P_{i}$ by $P_{j}$, is defined by

$\boldsymbol{F}_{i, k}=m_{i} \sum_{j=1 ; j \neq i}^{n}\left\{m_{j}\left(-\frac{p \sum_{\xi=0}^{\alpha-2}\left(r_{i j, k}^{\xi} r_{i j, k+1}^{\alpha-\xi-2}\right)}{r_{i j, k}^{\alpha-1} r_{i j, k+1}^{\alpha-1}\left(r_{i j, k+1}+r_{i j, k}\right)}\right.\right.$

$$
\left.\left.+\frac{q \sum_{\xi=0}^{\beta-2}\left(r_{i j, k}^{\xi} r_{i j, k+1}^{\beta-\xi-2}\right)}{r_{i j, k}^{\beta-1} r_{i j, k+1}^{\beta-1}\left(r_{i j, k+1}+r_{i j, k}\right)}\right)\left(\boldsymbol{x}_{i, k+1}+\boldsymbol{x}_{i, k}-\boldsymbol{x}_{j, k+1}-\boldsymbol{x}_{j, k}\right)\right\} .
$$


In particular, (4.1) defines discrete Newtonian gravitation when $p=G, q=0, \alpha=2$, while it defines a force with discrete Lennard-Jones potential when $\alpha=7, \beta=13$.

5. Conservation of energy. Consider now formula (2.5) with force defined by (4.1). In this connection the following lemma will be of value.

Lemma. For $n \geqq 2$, the following identity is valid:

$$
\begin{aligned}
& \sum_{j=1 ; j \neq i}^{n} \sum_{i=1}^{n} {\left[\begin{array}{c}
\frac{m_{i} m_{j} \sum_{\xi=0}^{\alpha-2}\left(r_{i j, k}^{\xi} r_{i j, k+1}^{\alpha-\xi-2}\right)}{r_{i j, k}^{\alpha-1} r_{i j, k+1}^{\alpha-1}\left(r_{i j, k+1}+r_{i j, k}\right)} \\
\left(\boldsymbol{x}_{i, k+1}+\boldsymbol{x}_{i, k}-\boldsymbol{x}_{j, k+1}-\boldsymbol{x}_{j, k}\right) \cdot\left(\boldsymbol{x}_{i, k+1}-\boldsymbol{x}_{i, k}\right)
\end{array}\right] } \\
& \equiv \sum_{i, j=1 ; i<j}^{n}\left\{m_{i} m_{j}\left(\frac{r_{i j, k+1}^{\alpha-1}-r_{i j, k}^{\alpha-1}}{r_{i j, k}^{\alpha-1} r_{i j, k+1}^{\alpha-1}}\right)\right\} .
\end{aligned}
$$

PROOF. The proof follows readily by mathematical induction on $n$. From (2.5), (4.1), and (5.1), then,

$$
\begin{aligned}
W & =\sum_{k=0}^{N-1} \sum_{i, j=1 ; i<j}^{n}\left\{m_{i} m_{j}\left(-p \frac{r_{i j, k+1}^{\alpha-1}-r_{i j, k}^{\alpha-1}}{r_{i j, k}^{\alpha-1} r_{i j, k+1}^{\alpha-1}}+q \frac{r_{i j, k+1}^{\beta-1}-r_{i j, k}^{\beta-1}}{r_{i j, k}^{\beta-1} r_{i j, k+1}^{\beta-1}}\right)\right\} \\
& =\sum_{i, j=1 ; i<j}^{n}\left\{m_{i} m_{j}\left[-p\left(\frac{1}{r_{i j, 0}^{\alpha-1}}-\frac{1}{r_{i j, N}^{\alpha-1}}\right)+q\left(\frac{1}{r_{i j, 0}^{\beta-1}}-\frac{1}{r_{i j, N}^{\beta-1}}\right)\right]\right\} .
\end{aligned}
$$

Defining the potential energy $V_{i j, k}$ of the pair $P_{i}$ and $P_{j}$ at time $t_{k}$ by

then yields

$$
V_{i j, k}=\left(-p / r_{i j, k}^{\alpha-1}+q / r_{i j, k}^{\beta-1}\right) m_{i} m_{j}
$$

$$
W=\sum_{i, j=1 ; i<j}^{n} V_{i j, 0}-\sum_{i, j=1 ; i<j}^{n} V_{i j, N} .
$$

Defining the potential energy $V_{k}$ of the system at $t_{k}$ by

$$
V_{k}=\sum_{i, j=1 ; i<j}^{n} V_{i j, k}
$$

then implies

$$
W=V_{0}-V_{N} .
$$

Finally, elimination of $W$ between (3.4) and (5.2) yields

$$
K_{N}+V_{N}=K_{0}+V_{0}, \quad N=0,1,2, \ldots,
$$

which is the classical law of conservation of energy.

6. Remarks. Let us note first that the algebraic formulation of this paper 
yields other basic classical results. With regard to initial value problems, for example, (2.3) and (4.1) imply

$$
\sum_{i=1}^{n} m_{i} \boldsymbol{a}_{i, k}=\mathbf{0},
$$

so that, from (2.2),

$$
\sum_{i=1}^{n} m_{i}\left(\boldsymbol{v}_{i, k+1}-\boldsymbol{v}_{i, k}\right)=\mathbf{0} .
$$

Summing both sides of (6.2) over $k$ from 0 to $s-1$, where $s \geqq 1$, yields

$$
\sum_{i=1}^{n} m_{i}\left(\boldsymbol{v}_{i, s}-\boldsymbol{v}_{i, 0}\right)=\mathbf{0} .
$$

Since (6.3) is valid also for $s=0$, then

$$
\sum_{i=1}^{n} m_{i} \boldsymbol{v}_{i, s}=c_{1}, \quad s \geqq 0,
$$

where $c_{1}$ is a constant vector. Formula (6.4) is, of course, the law of conservation of linear momentum. From (6.4) it then follows that

$$
\sum_{i=1}^{n} m_{i}\left(\frac{\boldsymbol{v}_{i, s+1}+\boldsymbol{v}_{i, s}}{2}\right)=\boldsymbol{c}_{1}, \quad s \geqq 0 .
$$

Thus, from (2.1),

$$
\sum_{i=1}^{n} m_{i}\left(x_{i, s+1}-x_{i, s}\right)=(\Delta t) c_{1}, \quad s \geqq 0 .
$$

Summing both sides of (6.6) over $s$ from 0 to $r-1$, for $r \geqq 1$, implies

$$
\sum_{i=1}^{n} m_{i}\left(x_{i, r}-x_{i, 0}\right)=t_{r} c_{1} .
$$

However, (6.7) is valid also for $r=0$, so that

$$
\sum_{i=1}^{n} m_{i} \boldsymbol{x}_{i, r}=t_{r} \boldsymbol{c}_{1}+\boldsymbol{c}_{2},
$$

where $c_{2}$ is a constant vector. Finally, set $M=\sum_{i=1}^{n} m_{i}$ and let $X_{r}$ be the center of gravity of the system at time $t_{r}$. Then (6.8) implies

$$
M X_{r}=t_{r} c_{1}+c_{2},
$$

from which it follows that the motion of the centroid is linear.

Finally, note that analogous techniques ([3], [4]) yield the same results of this paper for discrete mechanical systems in which (2.2) is replaced by either 


$$
\left(a_{i, k+1}+a_{i, k}\right) / 2=\left(v_{i, k+1}-v_{i, k}\right) /(\Delta t)
$$

or

$$
\frac{1}{2}\left(3 a_{i, k}-a_{i, k-1}\right)=\left(v_{i, k+1}-v_{i, k}\right) /(\Delta t)
$$

\section{REFERENCES}

1. R. P. Feynman, R. B. Leighton and M. Sands, The Feynman lectures on physics. Vol. 1: Mainly mechanics, radiation, and heat, Addison-Wesley, Reading, Mass., 1963. MR 35 \# 3942.

2. D. Greenspan, Introduction to numerical analysis and applications, Markham, Chicago, Ill., 1971. MR 42 \# 7028.

3. - New forms of discrete mechanics, Kybernetes 1 (1972), 87-101.

4. - A new explicit discrete mechanics with applications, TR 23, Computing Center, University of Wisconsin, Madison, Wis., 1971; J. Franklin Inst. 294 (1972), 231-240.

5. J. O. Hirschfelder, C. F. Curtiss and R. B. Bird, Molecular theory of gases and liquids, Wiley, New York, 1954.

Computer Science DePartment, University OF Wisconsin, Madison, Wisconsin 53706 